\title{
Ciudades y gobiernos locales: globalización, pobreza y democracia participativa
}

\author{
ALICIA ZICCARDI
}

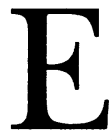

S DIFÍCIL ABORDAR EN UN BREVE ensayo cuáles son los principales problemas que deben enfrentar los gobiernos locales de las grandes ciudades latinoamericanas, tanto porque son innumerables cuanto porque los mismos suelen estar imbricados y se interpenetran. ¿Cuáles y cómo son los "ambientes" en los que operan las instituciones del gobierno local de las ciudades del siglo XXI? En este sentido, tratar de organizar una respuesta obliga a considerar por lo menos dos ejes de análisis: i) los procesos de globalización y competitividad económicas y los efectos que generan estas fuertes y rápidas transformaciones al incrementar la pobreza y la exclusión social y ii) los intentos de avanzar desde una democracia representativa a una democracia participativa como forma de gobierno de las ciudades. Éstos serán los dos ejes de análisis que intentaré desarrollar en este artículo.

\section{GLOBALIZACIÓN Y COMPETITIVIDAD URBANA}

Los estudiosos de las ciudades desde hace más de una década se han preguntado si existen las ciudades globales (Sassen, 1991) o si existen redes globales de ciudades (Borja y Castells, 1998). Pero en lo fundamental estas perspectivas coinciden en que, en la era de la información y del conocimiento, las ciudades son medios productores de innovación y riqueza y les cabe cumplir un papel estratégico como espacios articuladores de la economía nacional a la globalización. Es decir, las marcadas transformaciones que se advierten 
en las ciudades no sólo atañen a su fisonomía, sino a la naturaleza misma de las relaciones sociedad y territorio.

Richard Rogers (2000), desde su visión de urbanista, señala que allí donde solía haber dos grandes socios económicos (los negocios y los gobiernos nacionales) existe ahora un tercero: las ciudades globales. Más precisamente, las megalópolis que son aglomeraciones que ofrecen mano de obra con conocimientos y capacidades para operar tecnologías modernas y un conjunto de infraestructuras de localización y de comunicación que requiere la nueva red de ciudades globales.

En otro nivel de análisis, diferentes grupos de economistas han intentado hallar explicaciones sobre los nuevos vínculos que se han generado entre la economía y el territorio de la ciudad del siglo XXI, introduciendo para ello la noción de "competitividad urbana", la cual alude al proceso de generación y difusión de competencias que ofrecen las ciudades e incide en las capacidades de las empresas y las naciones para actuar exitosamente en un mundo globalizado.

Sin embargo, antes de introducir la idea de ciudades competitivas conviene aclarar que autores como Krugman (1996) consideran que las ciudades como tales no compiten unas con otras; son meramente el territorio (locus) de las empresas y firmas que son las que compiten; por lo tanto, desde esta perspectiva, las ciudades son una condición necesaria, pero no suficiente, para competir exitosamente.

Begg (2002) en cambio señala que, en contraste con otras épocas en las que las materias primas como los minerales o la tierra eran las principales fuentes de riqueza, hoy son las actividades urbanas la principal fuente de prosperidad económica. ${ }^{1}$ Por su parte Porter (1995, 1996), Lever y Turok (1999), Moori-Koening y Yoguel (1998) y Sobrino (2002) tienden a coincidir en que la competitividad es un proceso de generación y difusión de competencias el cual depende no sólo de factores de nivel micro, sino también de las capacidades que ofrece el territorio para facilitar las actividades económicas. Borja y Castells (1998) también coinciden en señalar que

${ }^{1}$ En México se estima que más de $85 \%$ de PIB proviene de las ciudades. 
las empresas no compiten aisladamente, sino que lo hacen junto con el entorno productivo e institucional de una ciudad o región urbana, mientras que Yoguel (2003) afirma que, cuando el sistema local funciona bien, el desarrollo de la capacidad innovadora de las firmas, y por lo tanto su competitividad, no dependen del tamaño de las empresas puesto que "el sistema local actúa como un cuasimercado que tiene un efecto palanca, aumentando las competencias técnicas y organizacionales de las firmas".

En síntesis, estos autores aceptan que es necesario crear en el espacio urbano un entorno físico, tecnológico, social, ambiental e institucional propicio para atraer y desarrollar actividades económicas generadoras de riqueza y empleo y, en este sentido, los gobiernos locales de las ciudades son los actores institucionales que pueden promover o generar estas condiciones y garantizar que el sistema educativo de nivel superior forme los recursos humanos especializados que demandan las nuevas actividades.

De acuerdo con estas posiciones, el papel que deben asumir los gobiernos locales como promotores del desarrollo económico va más allá de atraer la inversión, ya que también deben actuar para: i) crear un entorno institucional propicio; ii) elaborar regulaciones de calidad para normar los procesos de apropiación del suelo urbano; iii) impulsar la planeación estratégica del desarrollo local; $i v$ ) incorporar las TICs a las tareas de gobierno (por ejemplo, abrir páginas de la ciudad en internet); v) crear redes económicas e institucionales basadas en formas de cooperación y competencia para las pequeñas y medianas empresas (PYMES); vi) participar en instituciones y redes mediadoras e impulsoras de la cooperación entre los actores económicos que permiten a las ciudades o regiones urbanas competir en amplios mercados propios de una economía globalizada, como en las ciudades del Mercosur; vii) fomentar el vínculo entre las empresas que actúan en el medio local con las universidades y centros del enseñanza, a fin de que las tareas de investigación contribuyan a crear y difundir innovaciones y conocimientos.

2 Entre otros, véase Helmsing, 2002; Ferraro y Quintar, 1996; Vázquez Barquero, 2000 . 
Pero en los hechos, los gobiernos de las grandes ciudades latinoamericanas desarrollan estas tareas de manera incipiente y muchas de ellas ni siquiera se han asumido aún. ${ }^{3}$ Como contrapartida, puede advertirse que en estas ciudades la globalización y, quizá más concretamente, la aplicación de políticas neoliberales, han producido un fuerte proceso de desindustrialización y una rápida expansión de actividades del terciario moderno - comercio, turismo, servicios financieros - a partir de las cuales se intenta articular la economía nacional a la internacional, a la vez que se generan empleos para el relativamente pequeño contingente de los trabajadores que poseen mayor nivel educativo y capacitación. Pero la pérdida de puestos de trabajo en la industria y los servicios asociados a la misma ha implicado que para la mayoría de los trabajadores, que poseen un relativamente más bajo nivel educativo formal y de capacitación, las opciones ocupacionales se encuentren en el mercado de trabajo informal, de baja productividad, con bajos salarios y que no garantizan el acceso a la seguridad social.

\section{POBREZA Y EXCLUSIÓN SOCIAL}

Si bien es cierto que los gobiernos locales de nuestras ciudades deben asumir las tareas de transformar el espacio urbano con criterios más amplios de sostenibilidad económica, conservación del patrimonio histórico y cultural, y protección del medio ambiente, uno de los principales problemas que seguramente todos comparten y que se transforma en una prioridad a ser atendida por las políticas públicas es la pobreza y la exclusión social que deben aceptar estos trabajadores y sus familias para vivir en la gran ciudad. Sin duda, el gobierno local de las ciudades latinoamericanas tiene muchas dificultades para incidir directamente en el mercado de trabajo urbano, puesto que los gobiernos nacionales continúan siendo los que

${ }^{3} \mathrm{El}$ éxito de los distritos industriales de las ciudades europeas, particularmente italianas, así como los complejos territoriales de producción flexible de Estados Unidos llevaron a proponer en América Latina estrategias de competitividad basadas en la creación de una relación global-local creciente y armoniosa. Pero si bien este optimismo creó expectativas para que se desarrollaran regiones competitivas, Ferraro y Quintar (1996) han observado que en los hechos la realidad fue mucho menos integradora de lo que se esperaba. 
centralizan el diseño y la aplicación de las políticas económicas. Sin embargo, son numerosos los efectos urbanos que debe enfrentar como consecuencia de la expansión de estas actividades informales y precarias que realizan los trabajadores en el interior de las viviendas, en locales no habilitados o en las calles de las ciudades. Entre éstos deben mencionarse los que genera el llamado comercio ambulante, que confronta a las autoridades locales con diferentes sectores de la ciudadanía, pues lo que está en juego es el ejercicio del derecho al trabajo contra el ejercicio del derecho a disfrutar del espacio público.

La magnitud del problema es realmente alarmante; CEPAL (2003) estima que en 2002 el número de pobres en América Latina es de 221.4 millones, de los cuales 146.7 viven en el medio urbano. Asimismo señala que, aunque las mujeres han alcanzado en la región niveles de escolaridad superiores a los de los hombres, y las que forman parte de la población activa tienen en promedio más años de instrucción, son las principales víctimas del desempleo, la discriminación salarial y la escasez de tiempo. Es decir, la pobreza y la indigencia afecta más a las mujeres que a los hombres.

Se asiste a un problema de urbanización de la pobreza, es decir que el número de pobres urbanos respecto del total es cada vez mayor, y lo es también el número de familias de trabajadores que habitan en las megalópolis latinoamericanas en asentamientos periféricos y poseen una vivienda precaria en donde acceden a bienes y servicios colectivos de baja calidad (agua, drenaje, equipamientos de salud, educativos, culturales, recreativos, transporte). Sin duda todos éstos son claros indicadores de que predominan en el medio urbano situaciones de pobreza relativa. ${ }^{4}$ Pero a ello se agregan fuertes desigualdades sociales y diferentes formas de exclusión social, ${ }^{5}$ tales como las prácticas discriminatorias por género o etnia -que afectan

${ }^{4} \mathrm{El}$ concepto de pobreza absoluta que tiende a predominar en el medio rural hace referencia a que los individuos o las familias no tienen garantizados los niveles básicos de la sobrevivencia física. Sobre la distinción entre pobreza absoluta, pobreza relativa y exclusión social, véase entre otros Barnes et al., 2002.

${ }^{5} \mathrm{La}$ noción de exclusión social que ha sido acuñada por la sociología francesa alude a procesos sociales vinculados con la discriminación por género, etnia, lugar de vivienda, etc. a que son sujetos diferentes colectivos sociales. Sobre los nuevos retos que enfrentan las políticas públicas frente a los procesos de exclusión social, véase entre otros Brugué, Gomá y Subirats, 2002. 
a diferentes colectivos sociales- a las que se suman los obstáculos para acceder a la justicia, el crédito, los servicios básicos o la vivienda. Por ello debe considerarse que la polarización social que existe en la sociedad urbana latinoamericana en la actualidad contribuye a generar un clima propicio para que se desarrolle la inseguridad y la delincuencia hasta niveles nunca antes alcanzados, cuestión que deben enfrentar los gobiernos locales sin tener a veces las competencias plenas y los recursos adecuados para actuar con eficacia, aunque se trate de la primera y principal demanda de la ciudadanía.

Así, en las megalópolis de América Latina como São Paulo, México o Buenos Aires, los procesos de modernización económica vinculados a las actividades que promueve la globalización suelen localizarse en la ciudad central, la cual se transforma en una ciudad de servicios, mientras la industria manufacturera que logra sobrevivir a la competencia internacional se desconcentra hacia la periferia, que presenta una creciente sobrepoblación viviendo en condiciones paupérrimas, o hacia ciudades medias del interior del país que tienden a reproducir este mismo patrón de urbanización. Desde la perspectiva del ejercicio de gobierno, éste es sumamente complejo, porque el número de autoridades electas democráticamente por la ciudadanía es muy abultado y sobre todo por la inexistencia de formas de coordinación metropolitana eficaces entre las autoridades locales de los diferentes ámbitos de gobierno (federal, estatal o provincial y municipal) que actúan sobre estos extensos territorios urbanos. ${ }^{6}$

Lo limitado del presupuesto de los gobiernos locales, en gran medida destinado a gastos de administración de la ciudad consolidada, impide la formulación de políticas tendientes a construir obras públicas básicas, a lo que se agrega en algunos casos la imposibilidad de hacerlo por lo inapropiado del terreno en donde se han localizado originalmente estos barrios populares. Por ello otro de los rasgos más distintivos de las ciudades es el grave déficit de servicios y equipamientos básicos que presentan los barrios populares, los cuales en

${ }^{6}$ En las ciudades de México y Buenos Aires, por tratarse de ciudades capitales que forman parte de una zona metropolitana, esta gestión es aún más compleja ya que existe un amplio número de autoridades responsables de este territorio: jefe del ejecutivo local, gobernador, presidentes municipales, miembros del Legislativo local y provincial o estatal. 
algunas ciudades son verdaderas ciudades de pobres en el interior de la gran ciudad y constituyen una clara expresión espacial de una ciudadanía restringida.

Todos estos procesos, lejos de hacer de la ciudad un mecanismo de integración social, como lo sugirió Gino Germani (1967) hace ya más de tres décadas, hacen de las ciudades del siglo XXI ciudades fragmentadas, divididas o segmentadas, rasgos que las ciudades de América Latina siempre han tenido, pero actualmente se han amplificado (Ziccardi, 1995).

\section{DEMOCRACIA REPRESENTATIVA-DEMOCRACIA PARTICIPATIVA}

Aceptando que la democracia representativa, aunque imperfecta, es la mejor forma de gobierno, uno de los más importantes desafíos de las ciudades del siglo XXI es crear formas de relación cualitativamente diferentes y mejores entre representantes-representados, que contribuyan a que los gobiernos locales puedan mejorar, ser transparentes y respetar el estado de derecho en el ejercicio del gobierno político y la gestión urbana.

Se trata de que la ciudadanía no sólo ejerza sus derechos políticos a través del voto y luego delegue en los representantes de los partidos políticos la toma de decisiones, sino que se involucre en diferentes grados y etapas en el ejercicio de gobierno a fin de otorgar mayor eficacia a las decisiones públicas. En este sentido, la participación ciudadana es la inclusión de la ciudadanía en los procesos decisorios incorporando intereses particulares (no individuales), pero para que esto sea posible es necesario que existan nuevos espacios de participación que operen con reglas claras, las cuales favorezcan la deliberación pública, la interacción social y el respeto por el pluralismo político ( $c f$. Cunill, 1991; Ziccardi, 1998).

Durante muchas décadas, la acción gubernamental de las ciudades latinoamericanas se ha caracterizado por ser fuertemente centralizada, burocrática y autoritaria, así como poco eficaz en el ejercicio del gobierno. Hoy, muchas de estas características persisten, en tanto que los procesos de democratización - como los ocurridos en la ciudad de México o en Buenos Aires a finales de los años noventa - no han logrado aún revertirlas plenamente. Sin duda, más allá de la elección de los gobernantes locales por parte 
de la ciudadanía, lo que está en juego ahora es otorgar calidad a la democracia local, lo cual en el ejercicio de gobierno de una gran ciudad no sólo se define en el ámbito de la política, sino en el de la gestión urbana.

En el intento de democratizar la forma de gobierno, una cuestión fundamental es incorporar la participación de la ciudadanía en los procesos de decisión y gestión local. Si bien en la actualidad se registran muchos intentos por modificar las relaciones entre gobierno y ciudadanía, ${ }^{7}$ particularmente en el campo de las políticas sociales, se trata por lo general de experiencias puntuales que difícilmente puedan ser replicables y cuya consolidación tampoco está garantizada. $^{8}$ Es decir, la historia reciente confirma la idea de que, a pesar de que el municipio es la instancia de gobierno más próxima a la ciudadanía, las resistencias que operan en el espacio local para construir una cultura y prácticas democráticas no son fácilmente removibles para dar paso a la construcción de una gobernabilidad democrática que torne más eficaces y eficientes las políticas públicas.

Quienes gobiernan nuestras ciudades deben crear las condiciones para que sus habitantes sean ciudadanos, que puedan ejercer sus derechos políticos, sociales, culturales y urbanos cumpliendo con las obligaciones que implica habitar una ciudad. Aunque esto esté contenido en las leyes y reglamentos locales, no es suficiente si la sociedad local no es portadora de una cultura cívica para la vida comunitaria, la que por lo general deberá adquirirse en el mismo proceso de participación de la ciudadanía en los procesos decisorios. Para que ello ocurra es necesario abrir nuevos y mejores espacios de participación ciudadana en la gestión pública local, construir precisamente un espacio público en el que participe activamente la ciudadanía, tanto más cuando se trata de políticas sociales cuya principal finalidad es precisamente garantizar el ejercicio de la dimensión social de la ciudadanía. ${ }^{9}$ Es decir, debe existir una auténtica intención de parte de las fuerzas políticas y de la burocracia gubernamen-

${ }^{7}$ Cf. Ziccardi (coord.), 2004.

${ }^{8}$ Un conjunto de buenas experiencias de participación ciudadana en municipios mexicanos puede verse en Cabrero, 2002.

${ }^{9}$ Sobre las dimensiones de la ciudadanía véase el clásico texto de Marshall, 1998. 
tal para construir una gobernabilidad democrática en el ámbito local abriendo las compuertas a la participación ciudadana. Ante esta situación, la participación es revalorizada como un componente que puede otorgarle mayor calidad a la democracia porque puede mejorar el proceso de diseño y aplicación de las políticas públicas del ámbito local incorporando la presencia ciudadana. Por ello, el rescate de experiencias exitosas y las evaluaciones en torno a los resultados que se obtienen a partir de la inclusión de la ciudadanía en determinadas decisiones públicas es cada vez más frecuente.

Pero esto no es tarea fácil; por un lado, se advierte una gran complejidad en el aparato institucional y limitadas capacidades del personal de gobierno para atender las demandas y, por otro, una creciente demanda proveniente de los actores que forman parte de una cada vez más compleja sociedad local. Pero además es sabido que, en coyunturas de apertura o profundización de la democracia, las demandas de participación tienden a incrementarse, sobre todo por las expectativas que genera el cambio de régimen o la sola alternancia política entre partidos en el gobierno.

El gobierno local es un sujeto colectivo constituido por políticos, funcionarios, técnicos, personal administrativo, asesores, y deben definirse cuáles son los ámbitos institucionalizados y no instituciona$\operatorname{lizados}^{10}$ de participación, así como los instrumentos que existen para que la ciudadanía participe en los asuntos y en las decisiones públicas. La participación institucionalizada es precisamente aquella que está reconocida en las leyes y reglamentos de la ciudad y para que funcione debe incluir el diseño de las formas, los instrumentos y las reglas del juego. Pero ello no basta; sin duda hay que construir una relación de respeto mutuo y de atención a la participación social autónoma, creando también espacios de corresponsabilidad y actuación público-privados.

Hemos señalado en otro trabajo ( $c f$. Ziccardi, 2003) que las principales funciones de la participación ciudadana son: otorgar legitimidad al gobierno, ser un escalón en la construcción de una cultura democrática y contribuir a hacer más eficaces la decisión y gestión públicas. De igual forma, al analizar la incorporación de la parti- 
cipación de la ciudadanía en los procesos decisorios hemos distinguido analíticamente entre a) las formas o espacios de participación y b) los instrumentos de participación ciudadana. Conviene reiterar que, si bien ambos componentes de la participación están íntimamente relacionados, analizarlos separadamente puede contribuir a ofrecer más claridad y contribuir a lograr diseños participativos más eficaces.

En relación con los espacios de participación ciudadana, un elemento que el gobierno local debe resolver de manera adecuada es el tema de la representación, porque en las grandes ciudades es frecuente advertir un claro déficit de representatividad social, es decir, los diferentes sectores de la sociedad local - los jóvenes, las minorías indígenas, los discapacitados e inclusive las mujeres- no están plenamente representados. Respecto a esto último, en muchos partidos políticos los criterios de equidad de género funcionan en forma deficitaria, aun cuando debe reconocerse que es en los gobiernos de las grandes ciudades latinoamericanas donde existen actualmente mayores posibilidades de incrementar la presencia de mujeres en cargos de representación social y política, así como también en puestos de decisión del ejecutivo local. ${ }^{11}$

Los principales sustentos de la identidad grupal en las ciudades son el territorio (vecinos), la condición socio-económica (jóvenes, mujeres, empleados, obreros, trabajadores informales, etc.) y el interés por temáticas de la vida urbana y social (medio ambiente, vivienda, servicios, cultura, seguridad pública, etc.). De esta manera, en las ciudades existe una amplia variedad y gran número de asociaciones y agrupaciones de base territorial o social, muchas de las cuales están vinculadas con ONG tales como las de madres de familia con demandas de alimentos o por el acceso y calidad en la educación o la salud; las de colonos que piden la regularización de sus tierras, la construcción y/o mejoramiento de sus viviendas, la introducción de servicios; las organizaciones vecinales que reivindican

${ }^{11}$ En México, las alcaldesas en la última década han representado entre $3 \%$ y $4 \%$ del total de las presidencias municipales (menos de 100 en un total de 2429 municipios) en un contexto de profundas y rápidas transformaciones en el sistema político e importantes avances democráticos. En cambio, en países como Argentina y Brasil, esta relación ha mejorado sustancialmente. 
seguridad pública, calidad de vida, protección del patrimonio arquitectónico y cultural, respeto a la normatividad en los usos del suelo, mantenimiento de los espacios públicos y de las calles de la ciudad, entre muchas otras cosas.

Pero debe reconocerse que en las ciudades latinoamericanas la forma de concebir la participación que se hereda del estado corporativo exige su superación como requisito para democratizar la gestión pública. Antes de los años noventa, sólo en momentos excepcionales de fuertes crisis sociales como la generada en la ciudad de México a consecuencia de los sismos de 1985 se quebraron las reglas del juego clientelar y se abrió la participación a la ciudadanía afectada para incluir sus intereses y demandas en el proceso de reconstrucción de las viviendas ( $c f$. Mecatl, Michel y Ziccardi, 1987). En la actualidad, en cambio, los gobierno locales enfrentan de manera permanente los reclamos de una ciudadanía que actúa autónomamente, lo cual ha llevado a reconocer que la presencia del llamado capital social ${ }^{12}$ puede imprimirle a la tarea de gobernar mayor eficiencia administrativa y más eficacia política, siempre que se logre construir una efectiva relación de corresponsabilidad entre las instancias de gobierno y las organizaciones o agrupaciones de la sociedad civil.

En cuanto a los instrumentos de participación ciudadana, Joan Font (2001) afirma que el catálogo de instrumentos participativos no deja de crecer y su extensión, aunque desigual y limitada, también sigue una clara pauta ascendente. Esto surge claramente del inventario y análisis realizado sobre la experiencia europea y en particular catalana, así como también latinoamericana ( $c f$. Subirats et al., 2001). Pero también es cierto que los instrumentos de participación ciudadana son mucho más limitados en las ciudades latinoamericanas que en las europeas, tanto por su originalidad como por sus alcances, a excepción del presupuesto participativo creado por el Partido de los Trabajadores (PT) en la ciudad de Porto Alegre y aplicado ya en más de cincuenta ciudades brasileñas, latinoamericanas y españolas. El presupuesto participativo es realmente un instrumento original que combina la participación directa con la de-

12 Sobre la amplia y polémica noción de "capital social", véase el trabajo de Elinor Ostrom y T. K. Ahn, 2003. 
legación de responsabilidades en el personal gubernamental. Su principal objetivo es establecer las prioridades de la actuación pública local a través de la participación directa de la ciudadanías en reuniones y asambleas populares, las cuales se desarrollan con una metodología que permite ordenar y procesar las demandas. Los resultados forman parte del presupuesto municipal, el cual es liderado por un consejo de representantes de la ciudadanía y aprobado por la Cámara de Concejales (llamados vereadores en Brasil). Posteriormente, se elabora un plan de inversiones con los recursos disponibles que se aplica en el ejercicio del gobierno local. Otro instrumento que ha logrado modificar las relaciones gobierno-ciudadanía es la llamada planeación estratégica de las ciudades, ${ }^{13}$ proceso que se desarrolla con una metodología elaborada en Barcelona y que se ha aplicado ya para realizar los planes de desarrollo metropolitano de las ciudades de Buenos Aires, Córdoba, Bogotá, Quito y Río de Janeiro.

Pero en las ciudades latinoamericanas, los principales espacios de participación ciudadana son los consejos constituidos por expertos y/o miembros de la comunidad local a partir una representación social, territorial o sectorial, donde se trata por lo general de incorporar su opinión y construir consensos. Los instrumentos de participación ciudadana preferidos son los de consulta a la ciudadanía, tales como las encuestas y sondeos de opinión a través de los medios masivos o electrónicos, las audiencias públicas, etc. Sin embargo, estos procesos no siempre implican el diseño previo de una metodología de participación novedosa y adecuada a la realidad en la que se pretende funcionar. En muchos casos, son las mismas autoridades quienes suelen designar de manera directa o indirecta a los miembros de estos espacios de participación, no existen objetivos claros y/o el abultado número de miembros le resta efectividad. Todo esto convierte estos espacios en mecanismos de legitimación decisiones, no necesariamente públicas, sino con frecuencia exclusivamente gubernamentales.

${ }^{13}$ La concepción de planeación urbana estratégica fue elaborada por las ciudades que forman parte del Centro de Investigaciones del Desarrollo Urbano (CIDEU), asociación internacional de gobiernos locales promovida por el Ayuntamiento de Barcelona. 
Por todo lo expuesto, puede afirmarse que uno de los principales desafíos para construir una democracia participativa en las ciudades latinoamericanas es el de garantizar el ejercicio responsable de los derechos y las obligaciones de los ciudadanos. Pero tanto los funcionarios y los políticos del ámbito local como los ciudadanos deben compartir los valores de una cultura cívica basada en el respeto a la diversidad social y étnica, así como los propios del pluralismo político.

De las ideas expuestas quizá las más llamativas son las contradicciones o antagonismos que, con frecuencia están presentes en los problemas sociales. Así, en los procesos que inciden creando "el ambiente" más general en el que se desarrolla el ejercicio del gobierno local en las ciudades latinoamericanas, se advierte, por un lado, la intención de integrarlas a los procesos de globalización económica y crear condiciones de competitividad urbana, pero al mismo tiempo se advierte que la prioridad número uno es abatir la pobreza y exclusión social que genera el modelo económico y las políticas neoliberales adoptados. Por otro, en muchas ciudades existe la intención de los gobiernos locales de construir una democracia participativa, donde lo que existe es una frágil democracia representativa que funciona con un abultado y centralizado aparato de gobierno, a la vez que hereda unas relaciones entre gobierno y ciudadanía propias del modelo corporativo, signadas por el clientelismo político y el control social que prevaleció durante tantas décadas en las ciudades latinoamericanas.

\section{BIBLIOGRAFÍA}

Arce, Carlos, Enrique Cabrero y Alicia Ziccardi, comps. 2004. Ciudades del siglo XXI ¿Competitividad o cooperación? México: CIDE/COFEMER/Miguel Ángel Porrúa (en prensa).

Barnes, Matt et al. 2002. Poverty and Social Exclusion in Europe.: EE ed. Cheltenham-UK, Northamp-USA: Edward Elgar, 161 pp.

Begg, Iain. 1999. "Cities and Competitiveness". Urban Studies 36(5/6. mayo): 795-810.

Begg, Iain. 2002. "Urban Competitiveness". En Policies for Dynamic Cities, compilado por Iain Begg. Londres: The Policy Press, pp. 248. 
Bodemer, Klaus, José L. Coraggio y Alicia Ziccardi. 1999. "Políticas sociales urbanas al inicio del nuevo siglo". Cuaderno de Investigación. Montevideo: Unión Europea-Municipalidad de la Ciudad de Montevideo, $87 \mathrm{pp}$.

Borja, Jordi, y Manuel Castells. 1998. Local y global: la gestión de las ciudades en la era de la información. Madrid: United Nations for Human Settlements (UNCHS)/Taurus, $418 \mathrm{pp}$.

Boddy, Martin, "Linking Competitiveness and Cohesion". En Policies for Dynamic Cities, compilado por Iain Begg. Londres: The Policy Press, pp. 33-53.

Brugué, Quim, Ricard Gomá y Joan Subirats. 2002. "De la pobreza a la exclusión social. Nuevos retos para las políticas públicas”. Revista Internacional de Sociología 33 (septiembre-diciembre): 7-45.

Cabrero, Enrique, coord. 2003. Políticas públicas municipales. Una agenda en construcción. México: CIDE/Premio Gobierno y Gestión Local, 371 pp.

Castells, Manuel. 2000. "La ciudad de la nueva economía, La Factoría 12 (junio-septiembre) <www.lafactoria.com>.

Castells, Manuel. 1999. "La era de la información". Economía, Sociedad y Cultura, vol. 1: La Sociedad Red, Madrid: Alianza Editorial, 590 pp.

Coraggio, José Luis, S. Pintaudi et al. 1997. Globalización y competitividad metropolitana: grandes inversiones y reestructuración territorial. Buenos Aires: Geographikós.

Comisión Económica para América Latina (CEPAL). 2003. Panorama económico de América Latina. Santiago de Chile: CEPAL. <www.cepal.org>

Cunill, Nuria. 1991. La participación ciudadana. Caracas: Centro Latinoamericano de Administración para el Desarrollo (CLAD).

Fainsten, Susan, Ian Gordon y Michel Harloe. 1992. Divided Cities, New York and London in the Contemporary World. Oxford, Cambridge: Blackwell, 293 pp.

Ferraro, Carlo, y Aída Quintar. 1996. "Entornos territoriales activos. Relaciones de cooperación entre instituciones locales”. Buenos Aires: CEPAL, mimeo,

Fitoussi, Jean-Paul, y Pierre Rosanvallon. 1997. "La nueva era de las desigualdades". Buenos Aires: Manantial.

Font, Joan, coord. 2001. Ciudadanos y decisiones públicas. Barcelona: Ariel, 242 pp. 
Franco, Rolando. 1997. "Paradigmas de la política social en América latina". En Menjívar et al., pp. 35-59.

Germani, Gino. 1967. "La ciudad como mecanismo integrador". Revista Mexicana de Sociología 3(29) (julio-septiembre): 387-406.

Gomá, Ricard, y Óscar Rebollo. 2001. "Democracia local y ciudadanía activa: reflexiones en torno a los presupuestos participativos". En Ciudadanos y decisiones públicas, coordinado por Joan Font. Barcelona: Ariel, pp. 201-217.

Gordon, Ian. 1999. "Internationalisation and Urban Competition". Urban Studies 36(5/6): 1001-1016.

Helmsing, A. H. J. (Bert). 2002. "Perspectivas sobre el desarrollo económico localizado". Revista EURE 28(84), Santiago de Chile: 33-61.

Krugman, Paul. 1996. "Making Sense of the Competitiveness Debate”. Oxford Review of Economic Policy 12(3): 483-499.

Lever, William F., e Ivan Turok. 1999. "Competitive Cities: Introduction to the Review”. Urban Studies 36(5/6) (mayo): 791-794.

Marshall T. H. 1998. "Ciudadanía y clase social”. En Ciudadanía y clase social, compilado por T. H. Marshall y T. Bottomore. Madrid: Alianza Editorial, pp. 15-82.

Mecatl, José L., Marco A. Michel y Alicia Ziccardi. 1987. Casa a los damnificados. México: IISUNAM, $107 \mathrm{pp}$.

Menjívar, Larín et al. 1997. Pobreza, exclusión y política social. San José de Costa Rica: Flacso/Programa Most/Universidad de Utrech, 475 pp.

Moori-Koenig, Virginia, y Gabriel Yoguel. 1998. "El desarrollo de capacidades innovativas de las firmas en un medio de escaso desarrollo del sistema local de innovación”. Documento de trabajo, 9. San Miguel: Instituto de Industrias, UNGS.

Ostrom, Elinor, y T. K. Ahn. 2003. "Una perspectiva del capital social desde las ciencias sociales: capital social y acción colectiva". Revista Mexicana de Sociología 1 (enero-marzo): 155-233.

Porter, Michael. 1996. "Competitive Advantage, Agglomeration Economics, and Regional Policy”. Intermational Regional Science Review 19(1-2): 85-93.

Porter Michael. 1995. "The Competitive Advantage of the Inner City”. Harvard Business Review 73(3) (mayo-junio): 55-71.

Rogers, Richard. 2000. Ciudades para un pequeño planeta. Barcelona: Gustavo Gilli. 
Sassen, Saskia. 1998. "Ciudades en la economía global: enfoques teóricos y metodológicos". Revista EURE 24(71), Santiago de Chile (marzo): 5-25.

Sassen, Saskia. 1991. The Global City: New York, London, Tokyo. Princeton: Princeton University, $397 \mathrm{pp}$.

Sobrino, Jaime. 2002. "Competitividad y ventajas competitivas: revisión teórica y ejercicio de aplicación a 30 ciudades de México", Estudios Demográficos y Urbanos 17 (2).

Subirats, Joan et al. 2001. Experiencies de participació ciutadana en els municipis catalans. Barcelona: Generalitat de Catalunya.

Vázquez Barquero, Antonio. 2000. "Desarrollo endógeno y globalización". Revista EURE 26(79) Santiago de Chile (diciembre): 47-65.

Yoguel, Gabriel. 2003. "Algunas notas sobre desarrollo de sistemas locales y su influencia sobre las Pymes: el caso de Argentina”, LITTEC-UNGS, mimeo.

Yoguel y Boscherini. 2001. El desarrollo de las capacidades innovativas de las firmas y el rol del sistema territorial. Buenos Aires: Universidad Nacional del General Sarmiento.

Ziccardi, Alicia. coord. 1995. La tarea de gobernar. Gobiernos locales y demandas ciudadanas. México: IISUNAM/Miguel Ángel Porrúa, 399 pp.

Ziccardi, Alicia. 1998. Gobernabilidad y participación ciudadana en la ciudad capital. México: Miguel Ángel Porrúa/IISUNAM, 237 pp.

Ziccardi, Alicia. 2002a. "La demora de la democracia local: el difícil tránsito de vecinos a ciudadanos". Iberoamericana III(11) (septiembre), Instituto Ibero-Americano, Berlín: 161-177.

Ziccardi, Alicia, coord. 2003. Planeación participativa en el espacio local. Cinco Programas Parciales de Desarrollo Urbano en el Distrito Federal. México: IIS, PUEC y Posgrado de Urbanismo de la UNAM, 308 pp.

Ziccardi, Alicia, coord. 2004. Participación ciudadana y políticas sociales en el espacio local. Memoria. México: IISUNAM, Comecso, Indesol, 462 pp.

Instituto de Investigaciones Sociales, México, D.F. 